\section{Gl symptoms and fever increase the risk of severe illness and death in patients with COVID-19}

COVID-19 pandemic has become a major public health problem globally. Fever is the most common symptom. Besides fever, GI symptoms has also been reported in patients with COVID-19, such as diarrhoea, nausea, vomiting and abdominal pain and affect up to $28 \%$ of patients. ${ }^{1-5}$ Several previous studies assessed the association between GI symptoms and disease severity and yielded inconsistent results. ${ }^{1}{ }^{2}$ In this retrospective cohort study, we explored whether GI symptoms and fever increased the risk of severe illness or death in patients with COVID-19.

We analysed 29393 laboratoryconfirmed COVID-19 patients diagnosed before 21 March 2020 in cities outside of Wuhan in mainland China. The study was supported by the National Health Commission of China. The final data of follow-up were 17 April 2020. We excluded clinically diagnosed cases who were not laboratory confirmed and patients who were not discharged from hospital by 17 April 2020. The diagnosis of laboratory-confirmed COVID-19 case, severe cases (including critical cases) and death was conducted by local healthcare workers according to the national diagnosis and treatment protocol for COVID-19 released by the National Health Commission. ${ }^{7}$ The definition of having GI symptoms were patients with COVID-19 who had one of the following symptoms (nausea, vomiting, diarrhoea or abdominal pain). We used $\chi^{2}$ test or Fisher's exact test to compare the characteristics of four COVID-19 groups divided by GI symptoms and fever. Log-binomial regression models were used to estimate the crude and adjusted risk ratios (aRRs) and 95\% CIs of severe cases and death for patients with GI symptoms and fever, after adjusting for potential confounders. Two-sided $\mathrm{p}$ values of less than 0.05 were deemed to be statistically significant.

Characteristics of patients with COVID-19 with and without GI symptoms and fever are listed in table 1 . The median age was 47 years (IQR 34-57). The most frequent symptom was fever (69.9\%). Overall, 2289 (7.8\%) patients had GI symptoms. Five hundred and four (1.7\%) patients had GI symptoms and no fever, 18771 (63.9\%) patients had fever and no GI symptoms, 1785 (6.1\%) patients had both GI symptoms and fever 
Table 1 Characteristics of patients with COVID-19 with and without GI symptoms and fever

\begin{tabular}{|c|c|c|c|c|c|}
\hline \multirow[b]{2}{*}{ Characteristics } & \multicolumn{4}{|c|}{ COVID-19 patients groups by GI symptoms and fever (\%) } & \multirow[b]{2}{*}{$P$ value } \\
\hline & $\begin{array}{l}\text { No Gl symptoms or } \\
\text { fever }(n=8333)\end{array}$ & $\begin{array}{l}\text { Gl symptoms and no } \\
\text { fever }(n=504)\end{array}$ & $\begin{array}{l}\text { Fever and no } \\
\text { GI symptoms } \\
(n=18771)\end{array}$ & $\begin{array}{l}\text { Both GI symptoms } \\
\text { and fever }(n=1785)\end{array}$ & \\
\hline Age groups (years) & & & & & $<0.001^{*}$ \\
\hline$<30$ & $1765(21.2)$ & $57(11.3)$ & $2268(12.1)$ & $203(11.4)$ & \\
\hline $30-39$ & $1417(17.0)$ & $98(19.4)$ & $3746(20.0)$ & $385(21.6)$ & \\
\hline $40-49$ & $1511(18.1)$ & $72(14.3)$ & $4087(21.8)$ & $344(19.3)$ & \\
\hline $50-59$ & $1827(21.9)$ & $116(23.0)$ & $4599(24.5)$ & $420(23.5)$ & \\
\hline $60-69$ & $1070(12.8)$ & $86(17.1)$ & $2510(13.4)$ & $263(14.7)$ & \\
\hline $70-79$ & $547(6.6)$ & $59(11.7)$ & $1158(6.2)$ & $123(6.9)$ & \\
\hline$\geq 80$ & $196(2.4)$ & $16(3.2)$ & $403(2.1)$ & $47(2.6)$ & \\
\hline Sex (male) & $4021(48.3)$ & $195(38.7)$ & $10415(55.5)$ & $870(48.7)$ & $<0.001^{*}$ \\
\hline Region (outside of Hubei) & $4630(55.6)$ & $216(42.9)$ & $7560(40.3)$ & $688(38.5)$ & $<0.001^{*}$ \\
\hline History of chronic disease & $1814(21.8)$ & $171(33.9)$ & $4469(23.8)$ & $547(30.6)$ & $<0.001^{*}$ \\
\hline Exposure history (family or working place cluster) & $3637(47.3)$ & $172(37.9)$ & $4707(28.0)$ & $461(28.7)$ & $<0.001^{*}$ \\
\hline Shiver & $269(3.2)$ & $50(9.9)$ & $1980(10.5)$ & $296(16.6)$ & $<0.001^{*}$ \\
\hline Cough & $2381(28.6)$ & $186(36.9)$ & $6916(36.8)$ & $725(40.6)$ & $<0.001^{*}$ \\
\hline Expectoration & $1325(15.9)$ & $102(20.2)$ & $4288(22.8)$ & $503(28.2)$ & $<0.001^{*}$ \\
\hline Nasal obstruction & $343(4.1)$ & $29(5.8)$ & $784(4.2)$ & $102(5.7)$ & $0.005^{*} \dagger$ \\
\hline Runny nose & $359(4.3)$ & $43(8.5)$ & $978(5.2)$ & $139(7.8)$ & $<0.001^{*}$ \\
\hline Sore throat & $533(6.4)$ & $59(11.7)$ & $1532(8.2)$ & $183(10.3)$ & $<0.001^{*}$ \\
\hline Headache & $479(5.7)$ & 85 (16.9) & $2282(12.2)$ & $371(20.8)$ & $<0.001^{*}$ \\
\hline Dizziness & $67(0.8)$ & $8(1.6)$ & $183(1.0)$ & $39(2.2)$ & $<0.001^{*}$ \\
\hline Fatigue & $1148(13.8)$ & $139(27.6)$ & $4663(24.8)$ & $672(37.6)$ & $<0.001^{*}$ \\
\hline Muscle ache & $402(4.8)$ & $60(11.9)$ & $2116(11.3)$ & $396(22.2)$ & $<0.001^{*}$ \\
\hline Shortness of breath & $239(2.9)$ & $33(6.5)$ & $939(5.0)$ & $177(9.9)$ & $<0.001^{*}$ \\
\hline Dyspnoea & $185(2.2)$ & $37(7.3)$ & $716(3.8)$ & $165(9.2)$ & $<0.001^{*}$ \\
\hline Pneumonia feature by chest $\mathrm{X}$-ray/CT & $4908(58.9)$ & $407(80.8)$ & $16127(85.9)$ & $1568(87.8)$ & $<0.001^{*}$ \\
\hline Severe cases $\ddagger$ & $666(8.0)$ & $82(16.3)$ & $3332(17.8)$ & $369(20.7)$ & $<0.001^{*}$ \\
\hline Death & $93(1.1)$ & $15(3.0)$ & $533(2.8)$ & $70(3.9)$ & $<0.001^{*}$ \\
\hline
\end{tabular}

${ }^{*} \mathrm{P}<0.05$.

†Fisher's exact test.

flncluding critical cases.

and $8333(28.4 \%)$ patients did not have any GI symptoms or fever. The overall rate of severe cases and case fatality rate was $15.1 \%$ (95\% CI $14.7 \%$ to $15.5 \%$ ) and $2.4 \%$ (95\% CI $2.2 \%$ to $2.6 \%$ ), respectively. Compared with patients without GI symptoms or fever, patients with GI symptoms and no fever had a 50\% higher risk of severe illness (aRR 1.50, 95\% CI 1.14 to 1.97 ), patients with fever and no GI symptoms had a $81 \%$ higher risk of severe illness (aRR 1.81, 95\% CI 1.55 to 2.12 ), patients with both GI symptoms and fever had a $85 \%$ higher risk of severe illness (aRR 1.85, 95\% CI 1.67 to 2.04, all $\mathrm{p}<0.001$, figure $1 \mathrm{~A})$. Patients with GI symptoms and no fever did not have a significantly higher risk of death (aRR $1.73,95 \%$ CI 0.94 to 3.17 ), while patients with fever and no GI symptoms (aRR 2.04, 95\% CI 1.59 to 2.61) and patients with both GI symptoms and fever (aRR $2.43,95 \%$ CI 1.69 to 3.50 ) all had significantly higher risk of death (all $\mathrm{p}<0.001)$.
The associations were stable in the sensitivity analyses (model $\mathrm{A}$ and $\mathrm{B}$, figure $1 \mathrm{~B}$ ).

Jue Liu, ${ }^{1}$ Liyuan Tao, ${ }^{2}$ Xia Liu, ${ }^{3}$ Hongyan Yao, ${ }^{4}$ Shicheng Yu, ${ }^{4}$ Qiqi Wang, ${ }^{4}$ Jiaojiao Zhang, ${ }^{4}$ Zhancheng Gao, ${ }^{5}$ Rongmeng Jiang, ${ }^{6}$ Wenzhan Jing, ${ }^{1}$ Min Liu ${ }^{1}{ }^{1}$

${ }^{1}$ Department of Epidemiology and Biostatistics, School of Public Health, Peking University Health Science Centre, Beijing, China

${ }^{2}$ Research Center of Clinical Epidemiology, Peking University Third Hospital, Beijing, China

${ }^{3}$ Chinese Preventive Medicine Association, Beijing, China

${ }^{4}$ Chinese Center for Disease Control and Prevention, Beijing, China

${ }^{5}$ Department of Respiratory and Critical Care Medicine, Peking University People's Hospital, Beijing, China ${ }^{6}$ Centre for Infectious Disease, Capital Medical University Affiliated Beijing Ditan Hospital, Beijing, China

Correspondence to Professor Min Liu, Department of Epidemiology and Biostatistics, School of Public Health, Peking University Health Science Centre, Beijing 100191, China; liumin@bjmu.edu.cn

Acknowledgements We would thank all healthcare workers for their strong collaboration and great effects made in prevention and treatment of patients during COVID-19 outbreak and in collecting the information used in this study.

Contributors ML conceived the study, designed the study, collected the data, supervised the study, interpreted the results and revised the letter. $J \mathrm{~L}$ and LT searched the literature, analysed the data and wrote the letter. JL and LT contributed equally. JL, LT, $X L, H Y, S Y, Q W, J Z, Z G, R J$ and $M L$ revised the letter. WJ draw the figures. All authors contributed to the writing of the report. All authors contributed to writing the manuscript, and approved the final version for publication.

Funding This study was supported by the National Health Commission of China and funded by National Key Research and Development Project of China (2020YFC0846300), National Natural Science Foundation of China (71934002) and National Science and Technology Key Projects on Prevention and Treatment of Major Infectious Diseases of China (2020ZX10001002).

\section{Competing interests None declared.}

Patient and public involvement Patients and/ or the public were not involved in the design, or conduct, or reporting, or dissemination plans of this research.

Patient consent for publication Not required. 
A

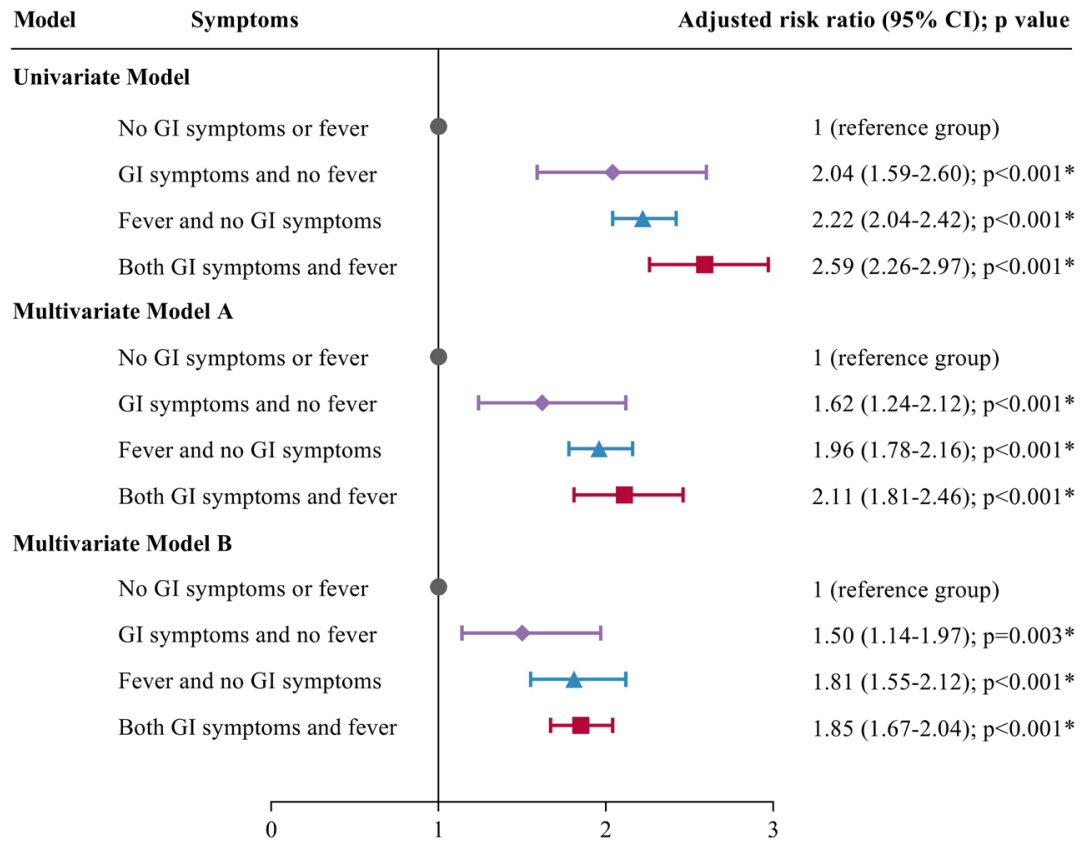

B

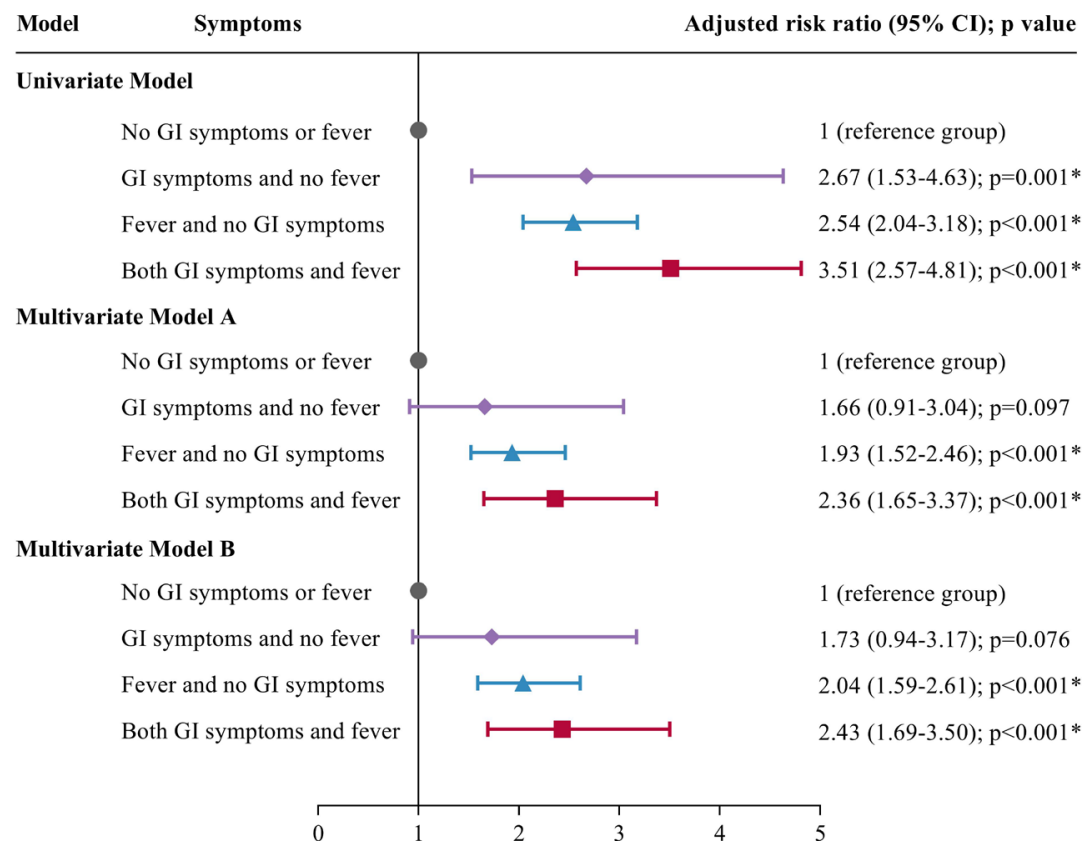

Figure 1 Associations of severe illness and death with Gl symptoms and fever in the univariate and multivariate models. (A) Risk ratios for severe illness; (B) risk ratios for death. In the univariate model, risk ratios were not adjusted for any confounders. In multivariate model $A$, risk ratios were additionally adjusted for age group, sex, region, history of chronic disease and exposure history of family or working place cluster. In multivariate model B, risk ratios were adjusted for onset symptoms (shiver, cough, expectoration, nasal obstruction, runny nose, sore throat, headache, dizziness, fatigue, muscle ache, shortness of breath and dyspnoea) and pneumonia feature by chest $\mathrm{X}$-ray/CT, in addition to the covariates in model $\mathrm{A}$.
Ethics approval The study was approved by the Institutional Review Board of Peking University(IRB00001052-20011).

Provenance and peer review Not commissioned; externally peer reviewed.

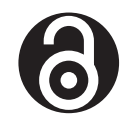

\section{OPEN ACCESS}

Open access This is an open access article distributed in accordance with the Creative Commons Attribution Non Commercial (CC BY-NC 4.0) license, which permits others to distribute, remix, adapt, build upon this work non-commercially, and license their derivative works on different terms, provided the original work is properly cited, appropriate credit is given, any changes made indicated, and the use is non-commercial. See: http:// creativecommons.org/licenses/by-nc/4.0/.

(C) Author(s) (or their employer(s)) 2021. Re-use permitted under CC BY-NC. No commercial re-use. See rights and permissions. Published by BMJ.

$J \mathrm{~L}$ and $\mathrm{LT}$ contributed equally.

\section{(A) Check for updates}

To cite Liu J, Tao L, Liu X, et al. Gut 2021;70:442-444. Received 8 May 2020

Revised 26 May 2020

Accepted 29 May 2020

Published Online First 30 June 2020

Gut 2021;70:442-444. doi:10.1136/

gutjnl-2020-321751

ORCID iD

Min Liu http://orcid.org/0000-0002-5059-3743

\section{REFERENCES}

1 Jin X, Lian J-S, Hu J-H, et al. Epidemiological, clinical and virological characteristics of 74 cases of coronavirusinfected disease 2019 (COVID-19) with gastrointestinal symptoms. Gut 2020;69:1002-9.

2 Lin L, Jiang X, Zhang Z, et al. Gastrointestina symptoms of 95 cases with SARS-CoV-2 infection. Gut 2020;69:997-1001.

3 Liang W, Feng Z, Rao S, et al. Diarrhoea may be underestimated: a missing link in 2019 novel coronavirus. Gut 2020:69:1141-3.

$4 \mathrm{Ng} \mathrm{SC}$, Tilg H. COVID-19 and the gastrointestinal tract: more than meets the eye. Gut 2020;69:973-4.

5 Effenberger M, Grabherr F, Mayr L, et al. Faecal calprotectin indicates intestinal inflammation in COVID-19. Gut 2020;69:1543-4.

6 Wang D, Hu B, Hu C, et al. Clinical characteristics of 138 hospitalized patients with 2019 novel CoronavirusInfected pneumonia in Wuhan, China. JAMA 2020:323:1061.

7 National Health Commission. Announcement on diagnosis and treatment protocol for novel coronavirus pneumonia, 2020. Available: http://www.nhc.gov.cn/ yzygj/s7653p/202003/46c9294a7dfe4cef80dc7f59 12eb1989.shtml 